\title{
A ATENÇÃO PRIMÁRIA À SAÚDE NOS CURSOS DE GRADUAÇÃO EM FISIOTERAPIA NO MUNICÍPIO DO RIO DE JANEIRO
}

\author{
PRIMARY HEALTH CARE IN THE PHYSIOTHERAPY UNDERGRADUATE \\ COURSES IN THE MUNICIPALITY OF RIO DE JANEIRO
}

LA ATENCIÓN PRIMARIA DE LA SALUD EN LAS CARRERAS DE

LICENCIATURA EN FISIOTERAPIA EN EL MUNICIPIO DE RIO DE JANEIRO

\author{
Nildo Campos Rangel Neto ${ }^{1}$ \\ Adriana Cavalcanti de Aguiar ${ }^{2}$
}

Resumo O estudo investigou como os cursos de graduação em Fisioterapia contemplam o ensino da Atenção Primária à Saúde no contexto da expansão da Estratégia Saúde da Família, tomando como referência as Diretrizes Curriculares Nacionais dos Cursos de Graduação em Fisioterapia. Foram analisados dez dos 14 cursos de Fisioterapia reconhecidos pelo Ministério da Educação e em funcionamento na cidade do Rio de Janeiro por ocasião da pesquisa. Entrevistamos os dez coordenadores e analisamos documentos relativos ao currículo e ensino. Os dados indicam que dois cursos estavam alinhados com as Diretrizes Curriculares por oferecerem atividades e/ou disciplinas e estágio supervisionado em Atenção Primária, com redistribuiçãoda carga horária do curso, atividades de práticas assistidas e de extensão. Sete cursos haviam implementado modificações curriculares parciais, sem no entanto incorporarem outras recomendações das Diretrizes Curriculares, especialmente a inclusão de disciplinas ou módulos de Atenção Primária à Saúde, não oferecendo oportunidades de formação em unidades de Saúde da Família. Um curso não havia implementado as Diretrizes. Os coordenadores mencionaram dificuldades para desenvolvimento curricular participativo. Concluímos que se verificam avanços significativos na incorporação da Atenção Primária à Saúde nos currículos estudados e discutimos alguns obstáculos na implementação das Diretrizes Curriculares Nacionais.

Palavras-chave fisioterapia; atenção primária a saúde; currículo.
Abstract The study investigated how the Physiotherapy undergraduate courses take into consideration the teaching of Primary Health Care within the context of the expansion of the Family Health Strategy, taking as reference the Brazilian National Syllabus Guidelines for Undergraduate Courses in Physiotherapy. We analyzed 10 out of the 14 Physiotherapy courses accredited by the Brazilian Ministry of Education that were being offered in the city of Rio de Janeiro at the time of the research. We interviewed the ten course coordinators and analyzed the documents regarding the teaching and the syllabus. The collected data indicate that two courses followed the Syllabus Guidelines by offering activities and/or disciplines and supervised internships in Primary Health Care, with a redistribution of the credit hours, and activities involving assisted practices and extension. Seven courses had implemented partial syllabus changes, but they did not incorporate other recommendations of the Syllabus Guidelines, especially the inclusion of Primary Health Care disciplines or modules, and did not offer training opportunities in Family Health units. One course had not implemented the Guidelines. The coordinators mentioned difficulties in developing a participatory syllabus. We concluded that we observed a significant progress in the incorporation of Primary Health Care in the analyzed syllabuses, and we discuss some of the obstacles in the implementation of the National Syllabus Guidelines.

Keywords physiotherapy; primary health care; syllabus. 


\section{Introdução}

Nos anos 1990 ampliou-se no Brasil a oferta de práticas em Fisioterapia em ambientes de Atenção Primária à Saúde (APS) (Brasil, 2006a), com o desafio de superar uma prática tradicional, tipicamente reabilitadora (Rebelatto e Botomé, 1999). Até então a formação universitária incluía poucos (ou nenhum) conteúdos afeitos ao tema, e excepcionalmente oferecia estágios em cenários de APS, o que não contribuiu para familiarizar os profissionais com as políticas de saúde contemporâneas.

Com a aprovação da Lei Orgânica da Saúde (lei n. 8080/90) foi criado o Sistema Único de Saúde (SUS), destacando-se, desde 1994, importante reforço da APS com o lançamento do Programa Saúde da Família (PSF), também conhecido como Estratégia Saúde da Família (ESF), com vistas a conferir resolutividade às ações do SUS e melhorar o estado de saúde da população, por meio de um modelo de assistência voltado à família e à comunidade, que abarca a promoção, identificação precoce, prevenção e tratamento das doenças (Brasil, 2008).

A territorialização informa o trabalho da ESF, e consiste na demarcação geográfica das áreas de atuação dos serviços, com reconhecimento do ambiente e dinâmica social local, e estabelecimento de relações estáveis em redes com outros serviços de saúde e equipamentos sociais (Gondim et al., 2001; Fonseca, 2007). A equipe 'básica' de Saúde da Família é formada por médico, enfermeiro, técnico de enfermagem e agentes comunitários de saúde (ACSs) (Brasil, 1998), fisioterapeutas atuam nos Núcleos de Apoio à Saúde da Família (NASFs), estabelecidos pela portaria ministerial n. 154/2008, visando agregar qualidade e resolutividade ao cuidado prestado (Brasil, 2004a; Brasil, 2008).

Na APS a contribuição potencial da Fisioterapia é grande: inclui, por exemplo, ações de promoção, prevenção, e atenção a agravos de alta prevalência como afecções do aparelho locomotor decorrentes do envelhecimento populacional. É de se esperar, portanto, que a atuação do fisioterapeuta na APS seja contemplada nos currículos da graduação. No entanto, muitos currículos de Fisioterapia no Brasil ainda sofrem a influência dos determinantes da Medicina, centrada no ambiente hospitalar como cenário principal de aprendizagem e formação (Bertoncello e Pivetta, 2015).

Esta lógica curricular ainda não incorpora os preceitos das Diretrizes Curriculares Nacionais (DCNs) para Cursos de Graduação em Fisioterapia nas quais o Conselho Nacional de Educação, após consultas públicas, estabeleceu

os princípios, fundamentos, condições e procedimentos da formação de fisioterapeutas (...) para aplicação em âmbito nacional na organização, desenvolvimento e avaliação dos projetos pedagógicos dos Cursos de Graduação em Fisioterapia das Instituições do Sistema de Ensino Superior (Brasil, 2002, art. 2, p.1). 
As DCNs flexibilizaram o desenvolvimento de currículos, em relação à legislação prévia, que estabelecia os chamados currículos mínimos (Brasil, 2002). Preconizam que fisioterapeutas tenham capacidade de "trabalhar em todos os níveis de atenção à saúde da população e de atender as necessidades da sociedade". Ao incorporarem um conceito ampliado de saúde e os princípios do SUS, as DCNs avançam na articulação entre Educação Superior e Saúde (Brasil, 1997;2001; 2009) e têm promovido mudanças no conteúdo dos currículos e nas metodologias de ensino-aprendizagem tornando-os mais generalistas e interferindo em relações de saber-poder e na prática docente (Signorelli et al., 2010).

A renovação das práticas de saúde demanda estratégias na formação profissional para induzir mudanças do processo de trabalho (Paim, 2002). Novas práticas implicam em novas competências, inclusive na compreensão integral do sujeito, da família e da comunidade, com consequências para a formação acadêmica (Ministério da Saúde, 2001; 2003; Oliveira, 2010), ao estabelecerem potencialmente um círculo virtuoso entre Educação e Trabalho, numa retroalimentação inovadora que deve ser incentivada.

Para atuar na ESF, o profissional precisa organizar, desenvolver e avaliar ações, além de articular recursos do território (Escorel, 2007; Fonseca, 2007). Esse modelo de atenção também demanda competências para atuar na promoção da saúde (Brasil, 2003; Gil, 2005), dificultada pela formação hegemônica hospitalocêntrica, fragmentada e pouco problematizadora (Feuerwerker e Sena, 2002; Besen et al., 2007; Oliveira, 2010). Programas de indução e iniciativas de Educação Permanente em Saúde (EPS), que compreendem a rede do SUS como espaço estratégico de desenvolvimento profissional têm sido adotadas e envolvemos cursos de graduação (Feuerwerker e Sena, 2002; Brasil, 2009). Implicam na interlocução entre os setores Saúde e Educação a fim de efetivar reformas curriculares que incluam a produção de conhecimentos no cotidiano das instituições de saúde (Ceccim e Ferla, 2005; Besen et al., 2007).

Apesar da sofisticada formulação de políticas públicas que articulam setores distintos, a formação do fisioterapeuta atualmente ainda se volta predominantemente para a reabilitação (Trelha et al., 2007; Bispo Junior, 2010), com pouca ênfase na compreensão ampliada do ser humano no contexto de suas relações sociais, conforme demanda a atuação na APS. Para tanto, o presente trabalho teve como objetivo investigar como os cursos de Graduação em Fisioterapia contemplam o ensino da APS no contexto da expansão da ESF, tomando como referência as DCNs dos Cursos de Graduação em Fisioterapia. Os cursos estudados estão localizados no município do Rio de Janeiro, que experimenta acelerada expansão da cobertura da ESF (de 3,5\% da população em 2009 para $44 \%$ até julho de 2014) (Brasil, 2014). 


\section{Métodos}

Esta investigação exploratória qualitativa buscou caracterizar o desenvolvimento dos currículos e a oferta dos cursos, sob a perspectiva dos gestores acadêmicos (coordenadores de curso) sobre o ensino da APS e implantação das DCNs. O instrumento empregado foi a entrevista individual presencial com roteiro semiestruturado com os dez coordenadores de curso (Fraser e Gondim, 2004). Também foi realizada análise dos documentos institucionais de domínio público disponível nas páginas institucionais na internet (estruturas curriculares e ementas das disciplinas) com o objetivo de embasar a discussão dos dados coletados (Minayo, 1996; 2004); no portal do Ministério da Educação (MEC) e documentos fornecidos pelos coordenadores de curso (projeto pedagógico, ementas de disciplinas ou módulos com conteúdos e/ ou práticas de APS).

O campo de pesquisa foi o município do Rio de Janeiro, onde funcionavam (de fato) 14 cursos de Fisioterapia (entre 16) autorizados pelo MEC por ocasião da coleta de dados. Por se tratar de um estudo sobre qualidade do ensino e inovação curricular, foram excluídos dois cursos com conceito menor que 2 no Exame Nacional de EnsinoSuperior (Brasil, 2007); um curso cujo gestortinha poucosmeses no cargo euma instituição de ensino superior (IES) cuja graduação em Fisioterapia havia iniciado há dois anos apenas. Portanto, participaram do estudo 10 cursos de Fisioterapia.

Para identificar as disciplinas ou módulos afins à APS foram selecionadas palavras-chave como: Saúde Pública, Saúde Coletiva, Fisioterapia Preventiva, Saúde da Família, Atenção Primária, Sistema Único de Saúde. De forma a fazer uma inclusão abrangente de disciplinas que busquem superar a fragmentação em órgãos e sistemas, também foram incluídas aquelas cujo recorte privilegia grupos populacionais e ciclos de vida. Tais palavras-chave foram buscadas tanto no enunciado das disciplinas quanto em seu conteúdo programático.

Foram entrevistados coordenadores dos dez cursos analisados, sendo abordados temas como a oferta de estágio em APS, os cenários em que os conteúdos de APS são oferecidos e as percepções dos coordenadores sobre os gestores em relação às DCNs e o desenvolvimento de currículos. As entrevistas ocorreram no fim de 2012, após os entrevistados assinarem o termo de consentimento livre e esclarecido (TCLE). O roteiro de entrevista foi pré-testado com um coordenador de curso de graduação da área de saúde a fim de minimizar ambiguidades do roteiro, no entanto não houve necessidade de mudança do mesmo. O contato com os coordenadores foi realizado por meio de mensagens eletrônicas e telefone, e as entrevistas realizadas em salas de coordenação nas IESs com duração média de 30 minutos. As entrevistas foram gravadas por um gravador digital da marca Sony. 
Após a caracterização dos entrevistados em relação ao tempo de formação, de docência e de coordenação do curso, foram abordados o currículo e as DCNs, e a formação em APS. Após a transcrição e organização, o material foi submetido à análise de conteúdo (Bardin, 2002). Foram estabelecidas as seguintes categorias analíticas: concepção de um novo currículo; novas metodologias de ensino-aprendizagem e atitudes de docentes e discentes; e práticas de ensino em APS.

A pesquisa recebeu aprovação do Comitê de Ética da Universidade Estácio de Sá sob o número 04324612.6.0000.5284 em 10/07/2012.

\section{Resultados}

Participaram do estudo cursos de dez IESs situadas no município do Rio de Janeiro (seis universidades, duas faculdades, um centro universitário e um instituto técnico), duas públicas e oito privadas, devidamente autorizadas para funcionamento. Apenas uma de cada IES foi escolhido aleatoriamente para esta pesquisa. Embora todos os entrevistados tenham recebido a mesma solicitação, apenas quatro coordenadores disponibilizaram os projetos pedagógicos de curso (PPC).

Para a análise dos resultados, os cursos e seus respectivos coordenadores foram numerados de 1 a 10 (de forma aleatória) para assegurar o anonimato das IESs, conforme acordado no TCLE.

\section{A concepção de um novo currículo}

Mudanças curriculares para atender as DCNs vêm sendo feitas por sete IESs participantes deste estudo. Duas outras começaram a oferecer o curso de Fisioterapia após a homologação das DCNs. Um dos cursos estudados não efetuou mudanças no currículo em função das Diretrizes em vigor.

Dentre as mudanças, houve redistribuição da carga horária $(\mathrm{CH})$ dos cursos; inclusão de novas disciplinas voltadas para a formação geral, contemplando os três níveis de atenção; estabelecimento de eixos curriculares afins à APS e aplicação de metodologias ativas, introduzindo alunos nos cenários de prática desde os primeiros períodos, o que inclui estágio curricular supervisionado dentro da ESF.

Os currículos vigentes são diversificados: alguns priorizam "metodologias mais ativas, com maior participação dos alunos e atenção integral com autonomia crescente nos níveis de atenção à saúde no decorrer do curso" (1), enquanto outros mantêm métodos de ensino-aprendizagem centrados no professor (baseados em aulas expositivas). A velocidade da mudança varia: 
três cursos adotaram as modificações no primeiro semestre de 2010, um o fez em 2012, e dois informaram que as modificações seriam implementadas em 2013.

Segundo o parecer CNE/CES n. 213/2008, a CH mínima recomendada é de 4 mil horas para o curso de graduação em Fisioterapia. Uma IES pesquisada não a contempla, e outra IES não oferece atividades práticas obrigatórias em APS, o que contraria o parecer CNE/CES n. 213/2008. Em relação às disciplinas afeitas ao ensino da APS, quase todas as IESs pesquisadas oferecem escassa $\mathrm{CH}$ quando comparadas aos outros níveis de atenção à saúde. Apenas três IESs oferecem disciplinas obrigatórias voltadas exclusivamente à APS, distribuídas por cinco períodos ou mais, e que são compreendidas como eixos curriculares. Uma (1) IES adotou 30\% de CH teórica de disciplinas com conteúdo de APS. Os demais cursos têm menos de $20 \%$ de $\mathrm{CH}$ destinada à temática da APS. Desses, quatro não atingiram nem 10\%. Oito cursos oferecem estágio supervisionado em APS, apesar do processo de trabalho do fisioterapeuta nestes cenários ainda ser pouco sistematizado, por exemplo, na definição da prática com as equipes de saúde da família.

De forma geral, a implementação de $\mathrm{CH}$ em APS está bem abaixo do preconizado para que o egresso esteja inserido no âmbito da reforma curricular (Gráfico 1).

\section{Gráfico 1}

Percentual da carga horária destinada às disciplinas nas Instituições de Ensino Superior

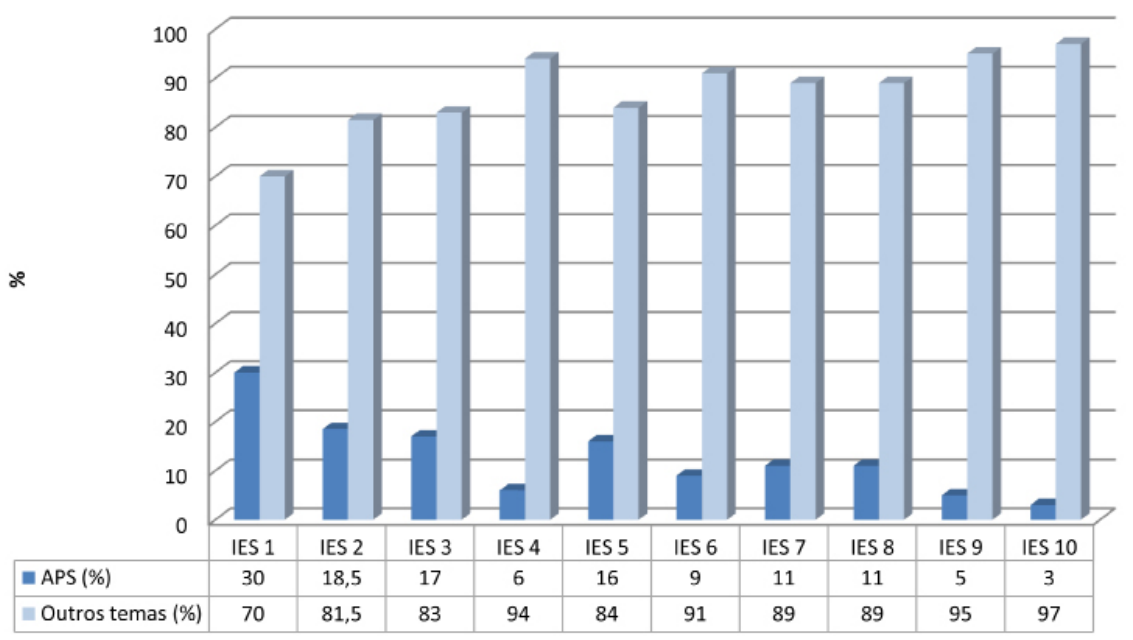

Fonte: Os autores.

Nota: Não foram computadas as disciplinas optativas.

Uma característica das DCNs é orientar as IESs a considerarem as necessidades do SUS na formação de recursos humanos, o que foi reiterado por todos os entrevistados: “a formação em fisioterapia deve, sem dúvida, estar voltada para a participação do egresso no SUS, pois é uma realidade” (3). 
Planejar e implementar um novo currículo não é fácil, segundo os coordenadores: "o currículo é vivo e precisa de constantes ajustes" (1). Segundo recomendação do MEC, oito IESs constituíram Núcleos Docente Estruturantes (NDEs), responsáveis pelas mudanças dos PPCs. Em uma IES os ajustes foram feitos por "todos os coordenadores, não só o de Fisioterapia" (6), contando com aportes da Comissão Própria de Avaliação (CPA). Um coordenador elaborou sozinho o PPC, e alegou que o corpo docente era "composto por professores horistas", o que significou que "só o coordenador tinha 40 horas semanais [contratuais], não havia muito com quem discutir" (9). Informou que após a criação do NDE o PPC foi revisto. Obstáculos institucionais para a implementação do novo currículo foram enunciados por dois coordenadores: ainda "existe toda uma questão de organização da faculdade que não dá para implementar tudo em termos administrativo e financeiro do processo" (2).

Na nova legislação, a duração mínima do curso passou para cinco anos (dez períodos), o que vigora em todos os cursos estudados, apesar da resistência dos discentes, segundo quatro coordenadores, pois "todo aluno quer se formar no menor tempo possível" (6).

Nove coordenadores identificam mudanças significativas em seus currículos após as DCNs. Por exemplo, a integração dos conteúdos, com o objetivo de "organizar melhor a vida acadêmica do aluno e permitir uma melhor flexibilização curricular (pesquisa, extensão, disciplinas optativas)" (1). Foi mencionado que a ampliação do curso permitiu organizar atividades extracurriculares e práticas supervisionadas, por meio do aumento do estágio curricular. O coordenador da IES 2 entende que a programação de novas disciplinas "englobou todas as competências específicas e também gerais da fisioterapia, a questão da APS, das metodologias ativas, passando para um currículo mais integrador" (2). No entanto, outro entrevistado observa que tantas modificações são difíceis de assimilar, porque "quando você concebe o curso, concebe a matriz, você imagina que vai acontecer de uma forma, e na implantação você identifica os erros (...) a falta de participação dos professores na implementação, a resistência discente" ${ }^{\prime \prime}(1)$. Três coordenadores afirmaram que a avaliação das novas abordagens curriculares tem sido realizada através das reuniões do NDE e da CPA (1,2 e 4).

Sobre o papel dos discentes nas mudanças, nove coordenadores afirmaram a importância de o aluno perceber a necessidade da APS como área de atuação. Para outro "o aluno deve passar por todo processo de atividades prevencionistas" (7). Para um dos entrevistados, a inclusão de novos eixos curriculares, como o 'eixo humanístico' (também incluído em outras duas IESs), é importante, pois eles apresentam para o aluno "o SUS, a introdução do que é e como funciona, a legislação, as partes mais aplicadas como a Saúde da Família (...), visualizando que a prevenção é o melhor caminho" (8). 
A humanização e resolutividade dos serviços e do sistema de saúde, que informam o currículo, foram apontados pelo coordenador da IES 1 como fatores para o desenvolvimento profissional do egresso, o que incluiu um eixo curricular sobre Educação e Promoção da Saúde, com disciplinas que tratam de APS em vários períodos.

Nas dez IESs analisadas, o ensino da APS abrange disciplinas de Saúde Coletiva; disciplinas que conectam Fisioterapia e APS e/ou que correlacionam diferentes níveis de atenção à saúde em torno de grupos populacionais, enfocando prevenção e reabilitação. Quanto à $\mathrm{CH}$ destinada a estas disciplinas, sete entrevistados consideram-na suficiente, três acham que é insuficiente; um argumentou que a APS não era prioridade no curso. Em duas matrizes curriculares, as disciplinas com APS aparecem em pelo menos oito dos dez períodos letivos. Ao compararmos a $\mathrm{CH}$ em APS com a com aquela das disciplinas práticas em outros níveis de atenção à saúde, observa-se que a mesma é reduzida. Um dos entrevistados afirmou: “o que a gente incluiu de APS a gente faz como atividades integradas e extensionistas" (10), implicando a extensão universitária como recurso para minimizar as lacunas do ensino.

\section{Habilidades e atitudes de docentes e discentes}

As DCNs demandam preparo pedagógico dos docentes, mas todos os entrevistados relataram dificuldades dos professores no ensino da APS. A falta de professores aptos a desenvolver as atividades em APS sofreria influência da formação tradicional: "os próprios docentes têm dificuldades em fazer o papel deles como fisioterapeutas, nos PSFs, imagina então em sala de aula" (4). Dois coordenadores afirmaram oferecer 'capacitações' para seus professores. Em um curso iniciado depois da homologação das DCNs, a capacitação docente foi precocemente iniciada: uma das IESs oferece incentivo à formação docente através de 'aulas pela internet' (3). Um dos entrevistados enfatizou que seus docentes "sempre procuram os encontros da Associação Brasileira de Ensino em Fisioterapia (Abenfisio)"(2).

Três coordenadores comentaram a importância de materializar metodologias ativas" com prevalência da problematização, a qual requer uma participação intensa do aluno" (1). Lançar mão da integração ensino-pesquisa foi uma opção, através das "disciplinas de práticas investigativas em que o aluno passa a ser o protagonista, e não mais o professor" (2). O coordenador da IES 3 afirmou que pelo menos uma disciplina por período apresenta atividade estruturada, que "é uma forma do aluno correlacionar a teoria com a prática através de visitas orientadas às instituições de saúde." A resistência por parte dos alunos a essa nova postura apareceu em seis entrevistas, sendo observado que alguns "percebem que a mudança é para melhor, principalmente na integralidade dos conhecimentos" (8), o que é facilitado pela participação 
em "fóruns discentes onde ocorre o diálogo com a coordenação em assuntos como ensino, extensão, pesquisa e infraestrutura" (3).

\section{Cenários e supervisão nas práticas da Atenção Primária à Saúde}

A complexidade dos processos saúde-doença-cuidado é percebida como algo que demanda novos métodos e cenários para o ensino-aprendizagem, "de forma a orientar o aluno para práticas mais prevencionistas" (3). Para dois coordenadores, a passagem pelo ambiente comunitário é fundamental: “a partir do momento que o aluno vê os problemas da comunidade, traz uma formação social para o aluno que se torna diferente" (4). Três IESs adotaram eixos temáticos que distribuem a APS por vários semestres. No entanto, sete gestores informaram que a ESF compõe o estágio supervisionado curricular, o qual sofre certo estranhamento por parte de alguns alunos, conforme mencionado por um coordenador. Apenas dois cursos têm $\mathrm{CH}$ em APS análoga à destinada aos outros níveis de atenção à saúde. Potenciais atividades a serem desenvolvidas pelos alunos na ESF foram descritas: "Dinâmicas de grupos, por exemplo: diabético, hipertenso, o grupo cuidando de quem cuida (os nossos alunos cuidando dos profissionais que atuam na ESF). Realizamos visitas domiciliares, orientações, grupos, (...) em conjunto ou não com os profissionais que ali atuam (2)."

Todos os coordenadores informaram problemas na oferta de estágio em APS: dificuldades em firmar convênios com o SUS no âmbito municipal, de inserir preceptores na ESF, dependência de disponibilidade de vagas na rede de serviços. Quanto à supervisão, um coordenador apontou como dificuldade encontrar "profissionais qualificados que possam ensinar a Fisioterapia na ESF" (5). Os alunos da IES 2 são acompanhados por um professor "em trabalho conjunto com o profissional que está no PSF" (2). Essa solução, no entanto, foi problematizada: "nós não podemos participar da assistência fisioterápica, pois há diversas contrapartidas, você tem que ter um profissional do local". Porém, um entrevistado observou que "os preceptores do SUS nem sempre estão dispostos e disponíveis para receberem alunos; esperamos que o MEC e o Ministério da Saúde conversem para que possamos ter condições de contar com estes preceptores (1)".

Uma prática controversa na tentativa de oferecer estágio em APS é apontada por um coordenador: a criação de cenários que mimetizem o ambiente comunitário: "tem um projeto interessante em estágio em APS, onde foi instituído o modelo do SUS na clínica escola para atenção básica" (5). Um curso oferece somente uma disciplina de Saúde Pública: “a ESF ainda não está no currículo, pois ainda não foi reformulado" (10). Curiosamente esse coordenador foi o único a mencionar que participa do Pró-Saúde 3, um programa interministerial de indução de mudanças na graduação. Outro coordenador 
citou parcerias entre instituições de ensino superior que ajudaram na inclusão dos alunos na temática ESF: "Realizamos atividades em conjunto com a Universidade $[\mathrm{X}]^{\prime \prime}(4)$.

Embora as DCNs determinem que o estágio curricular supervisionado deve assegurar a prática de intervenções preventiva e curativa nos diferentes níveis de atuação, na maioria das IESs isso não ocorre. Apenas três IESs pesquisadas realizam estágios na ESF; duas IESs já firmaram convênios com o município e informaram intenção de iniciar suas atividades em breve; quatro IESs apresentam 'estágio em APS'; no entanto, seus coordenadores reconhecem que a $\mathrm{CH}$ é mínima, enquanto em outras duas o estágio em APS não é obrigatório. Em alguns casos, embora atuem no cenário da APS, os alunos realizam apenas práticas reabilitadoras dentro da unidade de saúde.

\section{Discussão da inserção da Atenção Primária à Saúde no ensino da Fisioterapia}

Passados mais de dez anos da homologação das DCNs, a maioria dos conteúdos dos cursos investigados ainda enfocavam a atenção secundária/terciária, embora se observem mudanças no ensino de Fisioterapia no município do Rio de Janeiro. Ainda assim, na formação dos profissionais de fisioterapia predominava o modelo flexneriano, focadana reabilitação como eixo do ensino-aprendizagem (Souza, 2006; Bispo Junior, 2010).

A necessidade de mudanças na graduação das profissões de saúde e da busca de superação do modelo hospitalocêntrico demonstram algum reconhecimento da integralidade da atenção, mas iniciativas além das diretrizes ainda são necessárias para gerar mudanças mais profundas (Ceccim e Feuerwerker, 2004; Almeida, Campos e González apud Streit et al., 2012).

Os dados do presente estudo indicam que as DCNs impulsionaram algum grau de debate e formulação de novos currículos para a formação de fisioterapeutas no município do Rio de Janeiro, em graus diferenciados nos dez cursos estudados. Dois cursos aderiram mais às recomendações das DCNs e redistribuíram a carga horária das disciplinas, incluíram novas disciplinas voltadas para uma formação geral que contempla os três níveis de atenção à saúde e incorpora metodologias ativas de ensino-aprendizagem.

Algumas IESs avançaram mais e outras ainda estão se adequando à 'nova realidade' curricular e convivendo com lacunas que podem influenciar de forma significante a formação do egresso. Algumas dessas lacunas tinham sido preenchidas com a oferta de disciplinas de práticas assistidas em quatro dos dez cursos investigados.

Essa 'nova realidade' aponta que a formação dofisioterapeuta deve contemplar as necessidades sociais dasaúde conforme preconizado pelos princípios do SUS, o que não deve ser confundido apenas com aprendizagem da atenção básica, pois demanda integrar a atenção no âmbito dapromoção, proteção e 
recuperação da saúde (Gallo, 2005; Brasil, 2006b; Seriano, Muniz e Carvalho, 2013). Araújo, Miranda e Brasil (2007) observam que o SUS representa um grande mercado de trabalho, mas a formação do fisioterapeuta precisa contemplar o estímulo ao pensamento crítico, baseada nos problemas da população assistida, ea valorização do conhecimento cotidianamente produzido nas unidades de saúde, em articulação com o que é criado na universidade.

O ritmo variável de implantação de mudanças ocasiona um atraso na incorporação de conteúdos e práticas que permitam "inserir efetivamente o fisioterapeuta nos serviços de atenção primária à saúde, superando a visão do profissional voltado apenas para a reabilitação (...)" (Parecer CNE/CES n. 213/2008) (Bertoncello e Pivetta, 2015).

A inclusão de um número maior de disciplinas com conteúdos de APS em conjunto com a redistribuição da carga horária ainda se faz necessária para reforçar o ensino-aprendizagem nos currículos daquelas IESs que ainda não conseguiram se adequar às DCNs, fortalecendo a formação do egresso na busca de formação generalista (Escorel, 2007; Souza et al., 2014).

Apesar de a maioria dos cursos investigados contarem com um NDE, principal instância responsável pela construção dos projetos pedagógicos, foi mencionada a dificuldade de engajar o corpo docente através de reuniões dos colegiados dos cursos, pois uma grande parte dos professores é'horista', sem disponibilidade para reuniões, visto que na maioria dos casos as instituições eram privadas e regidas pela CLT. Por parte dos coordenadores, de apenas quatro PPC silustra a dificuldade de usar estes documentos como fontes de dados. O PPC deve nortear as atividades desenvolvidas pela IES e explicitar os seus compromissos na provisão do ensino, com base em sua concepção de educação e do perfil que espera do seu egresso. Foi observado um possível receio por parte dos coordenadores em fornecer os PPCs, com o argumento de que a direção da IES deveria autorizar sua liberação para a pesquisa.

Nenhum entrevistado mencionou o desenvolvimento de pesquisas em APS, o que atrasa a produção de conhecimento que possa nortear as atribuições do fisioterapeuta no âmbito da APS. No entanto, todos os entrevistados mencionam a existência de projetos de extensão voltados para a comunidade, ainda que no âmbito da reabilitação.

Um entrevistado afirmou que o seu curso inclui a APS em seu currículo como 'atividades extensionistas'. É importante discernir que tais atividades não compõem o currículo dos cursos nem são contempladas na carga horária total dos mesmos. A emergência, nessa entrevista, da extensão como oportunidade de prática da APS significa uma distorção do conceito, de acordo com o Fórum de Pró-Reitores de Extensão das Universidades Públicas Brasileiras (2012): 
A Extensão Universitária, sob o princípio constitucional da indissociabilidade entre ensino, pesquisa e extensão, é um processo interdisciplinar, educativo, cultural, científico e político que promove a interação transformadora entre Universidade e outros setores da sociedade.

A extensão universitária não compõe a matriz curricular como ensino ou pesquisa, embora, conforme a Constituição de 1988, denote prática acadêmica, a ser desenvolvida com o Ensino e a Pesquisa, visando à promoção e garantia dos valores democráticos, da equidade e do desenvolvimento da sociedade em suas dimensões humana, ética, econômica, cultural, social. Desta forma, a extensão engloba várias atividades com o intuito de promover a participação do aluno na prestação de serviços à sociedade. As atividades de extensão universitária não podem substituir conteúdos e práticas obrigatórios para a formação dos egressos conforme perfil preconizado.

A CH destinada ao estágio supervisionado curricular deve representar $20 \%$ do total de horas dos cursos de graduação em Fisioterapia, segundo a Câmara de Educação Superior do Conselho Nacional de Educação (resolução CNE/CES n. 4/2002). A resolução que dispõe sobre o assunto afirma que o estágio curricular supervisionado deverá assegurar a prática de intervenções preventiva e curativa nos diferentes níveis de atuação, no entanto. Não há nas DCNs um artigo que mostre como a $\mathrm{CH}$ total de estágio deve ser dividida entre os três níveis de atenção, porém, ao admitir-se que o egresso deve ter "uma formação generalista, humanista, crítica e reflexiva, capacitado a atuar em todos os níveis de atenção à saúde (...) respeitando os princípios éticos/ bioéticos, e culturais do indivíduo e da coletividade (resolução CNE/CES n. 4/2002)", a CH destinada às atividades de prática deveriam ser distribuídas de acordo com os níveis de complexidade de forma equivalente (Seriano, Muniz e Carvalho, 2013).

Para tanto, a $\mathrm{CH}$ destinada às atividades práticas demandariam redistribuição que permitisse maior inserção dos alunos no ambiente de APS, pois segundo Feuerwerker e Sena (2002) os cenários de prática estimulam a capacidade crítico-reflexiva, com foco na problematização e articulação dos diferentes atores sobre os problemas da realidade, o que favorece a construção coletiva do conhecimento e propicia a liberdade no pensar e de agir.

Todavia, diante do cenário identificado nesse estudo, a formação do fisioterapeuta enfrenta vários desafios, como o de viabilizar novos cenários de prática profissional para que os estudantes, sob supervisão adequada, aprendam a atuar fora do ambiente altamente especializado. O que prevaleceu foram conteúdos e práticas voltados para as especialidades e para a abordagem reabilitadora (Signorelli et al., 2010).

Um importante entrave para a oferta do estágio curricular em APS, conforme informado pelos coordenadores de cursos privados, foi a expectativa 
das instituições de saúde de obterem contrapartida financeira (remuneração ou bolsas de estudo) e de contarem com supervisores de estágio contratados pelas IESs, o que encontra resistência por parte das mantenedoras.

Além disso, todos os coordenadores relataram dificuldades por falta de docentes e preceptores aptos a aplicarem novas metodologias de ensinoaprendizagem e atuarem em cenários de APS. Sobre a supervisão no estágio curricular as DCNs estabelecem: "A formação do fisioterapeuta deve garantir o desenvolvimento de estágios curriculares, sob supervisão docente" (Brasil, 2003). Na prática, coexistem situações distintas de supervisão em APS: havia preceptores que eram profissionais da instituição conveniada; fisioterapeutas contratados pela IES alocados no cenário de prática (com restrição da margem de atuação);e docentes de IES(o que seria desejável para fortalecer a integração teoria-prática), porém poucos docentes estavam engajados de fato nos cenários. Isso dificulta a oferta do estágio em APS, principalmente no âmbito da ESF, situação agravada pela incipiente sistematização das atribuições do fisioterapeuta nesse tipo de cenário de prática, o que reforça a importância do investimento na preparação de docentes e preceptores (Silva e da Ros, 2007).

A Abenfisio e o FNEPAS (Fórum Nacional de Educação das Profissões na Área da Saúde) vêm atuando para fortalecer lideranças que facilitem as modificações necessárias para garantir a implantação das DCNs (Associação Brasileira de Ensino em Fisioterapia, 2012). Por outro lado, é fundamental que as equipes que estão nos NASFs, nos municípios, estados e IESs compreendam a relação entre formação, educação continuada e permanente e prática profissional. Os problemas em relação à preceptoria de estágio em ambientes de APS poderiam ser amenizados por meio do Programa de Educação pelo Trabalho para Saúde (PET-Saúde), que tem como objetivo a educação pelo trabalho, que oferece bolsas para tutores (professores) e preceptores (profissionais dos serviços), além de estudantes de graduação da área de saúde. Porém, durante as entrevistas, não surgiu nenhum comentário sobre a participação do curso no PET-Saúde (Costa et al., 2015).

\section{Considerações finais}

O presente estudo apontou uma renovação parcial dos currículos dos cursos de graduação em Fisioterapia do município do Rio de Janeiro na direção preconizada pelas DCNs. Algumas IESs estão mais adiantadas neste processo, outras ainda estão se adequando a atual realidade estabelecida nas DCNs.

O MEC pode dialogar com o MS ecom os governos estaduais e municipais e, junto com as IESs, pactuar melhor os mecanismos para fortalecer a implantação das DCNs, por exemplo, ao viabilizar parcerias que favoreçam o oferecimento de estágio curricular em APS na rede do SUS. Cabe uma normatização do vínculo dos preceptores e a reflexão sobre a natureza da supervisão docente para 
fortalecer a qualidade do estágio curricular. É interessante também articular o desenvolvimento da preceptoria com a Política Nacional de Educação Permanente (portaria n. 1.996 de 20 de agosto de 2007), de modo a direcionar a contribuição das IESs para o aprimoramento do processo de trabalho em APS.

Políticas de inclusão do fisioterapeuta nos NASFs foram elaboradas e implementadas, mas as atribuições do mesmo ainda não foram totalmente estabelecidas, o que dificulta o cuidado à população e a formação profissional. Os conhecimentos se renovam e os processos de trabalho são distintos em diferentes contextos de prática. A heterogeneidade de dinâmicas de funcionamento dos NASFs de acordo com distintas realidades locais pode dificultar a formulação de uma estratégia de ensino pelos cursos de Fisioterapia. Como a graduação não esgota a formação, cabe preparar o aluno na perspectiva da educação continuada e permanente.

Ainda que exista uma relação muito importante entre a prática e a formação profissional, a inovação na formação por si só não muda a prática e mudanças no mercado de trabalho e nas práticas não determinam necessariamente novos rumos para a formação. Os coordenadores entrevistados assinalam que o mercado de trabalho em APS ainda é escasso para fisioterapeutas, por maior que seja a necessidade de atenção fisioterápica em unidades do SUS. Por outro lado, os cursos podem preparar os egressos para uma atitude ativa de pressionar pela identificação e atenção às necessidades de saúde da população, fortalecendo a ESF, também fomente esta discussão.

É necessária a criação de mecanismos efetivos de avaliação por ocasião da autorização, do reconhecimento e da renovação do reconhecimento dos cursos, para que o MEC assegure a plena implementação das DCNs. Os documentos utilizados pelos avaliadores do MEC podem demandar o detalhamento dos cenários de prática e a relação entre ensino, pesquisa e extensão em APS, e examinar em detalhe a preceptoria do estágio, aspectos vitais para mudança na formação e, indiretamente, construção da integralidade na prática. O fato de os PPCs dos cursos não serem de domínio público impede a transparência do processo e do acesso aos currículos e não norteia futuros interessados em cursar a Fisioterapia.

Em IESs majoritariamente privadas, várias com fins lucrativos, muitos professores têm vínculos precários e $\mathrm{CH}$ contratual restrita, o que restringe a possibilidade de discussão e formulação curricular coletiva e enfraquece os PPCs na prática. Por fim, ainda que não tenham sido objeto do presente trabalho, é necessário problematizar as práticas de avaliação formativas e somativas dos alunos, à luz do perfil de competência estabelecido, de modo a avançar no desenvolvimento de ferramentas e estratégias de avaliação das ações realizadas na interface entre academia e serviços. 


\section{Colaboradores}

Nildo Campos Rangel Netoe Adriana Cavalcanti de Aguiar são igualmente responsáveis pela concepção do estudo, elaboração de instrumentos, coleta e análise de dados e redação do artigo. Não há conflitos de interesses.

Resumen El estudio investigó cómo las carreras de licenciatura en Fisioterapia contemplan la enseñanza de la Atención Primaria de la Salud en el contexto de la expansión de la Estrategia de Salud de la Familia, tomando como referencia las Directrices Curriculares Nacionales de las Carreras de Licenciatura en Fisioterapia. Se analizaron diez de las 14 carreras de Fisioterapia reconocidas por el Ministerio de Educación y en vigor en la ciudad de Rio de Janeiro durante el período de la investigación. Entrevistamos a los diez coordinadores y analizamos documentos relativos al plan de estudios y la enseñanza. Los datos indican que dos de los cursos estaban alineados con las Directrices Curriculares por ofrecer actividades y/o disciplinas y práctica/residencia en Atención Primaria, con redistribución de la carga horaria del curso, actividades de prácticas asistidas y de extensión. Siete carreras implementaron modificaciones curriculares parciales pero sin incorporar otras recomendaciones de las Directrices Curriculares, especialmente la inclusión de disciplinas o módulos de Atención Primaria de la Salud, no ofreciendo oportunidades de formación en unidades de Salud de la Familia. Una carrera no implementó las Directrices. Los coordinadores mencionaron dificultades para el desarrollo curricular participativo. Concluimos que se observan avances significativos en la incorporación de la Atención Primaria de la Salud en los planes de estudio analizados y discutimos algunos obstáculos en la implementación de las Directrices Curriculares Nacionales. Palabras clave fisioterapia; atención primaria de la salud; planes de estudio.

\section{Notas}

${ }^{1}$ Universidade Estácio de Sá, Centro de Ciências Biológicas e da Saúde, Nova Iguaçu, RJ, Brasil.

$<$ nildorangel99@gmail.com>

Correspondência: Universidade Estácio de Sá, Centro de Ciências Biológicas e da Saúde, rua Oscar Soares, 1.466, Jardim Califórnia, CEP 26220-099, Nova Iguaçu, Rio de Janeiro, Brasil.

${ }^{2}$ Fundação Oswaldo Cruz, Instituto de Comunicação e Informação Científica e Tecnológica em Saúde, Rio de Janeiro, RJ, Brasil.

<adriana.aguiar@post.harvard.edu> 


\section{Referências}

ARAÚJO, Dolores; MIRANDA, Maria C. G.; BRASIL, Sandra L. Formação de profissionais da saúde na perspectiva da integralidade. Revista Baiana de Saúde Pública, Salvador, vol. 31, supl. 1, p. 20-31, 2007.

BARDIN, Laurence. Análise de conteúdo. Lisboa: Edições 70, 2002.

BERTONCELLO, Dernival; PIVETTA, Hedioneia M. F. Diretrizes curriculares nacionais para a graduação em fisioterapia: reflexões necessárias. Caderno de Educação, Saúde e Fisioterapia, Porto Alegre,v. 2, n. 4, p. 72-84, 2015.

BESEN, Candice B. et al. The family health strategy as object of health education. Saúde e Sociedade, São Paulo, v. 16, n. 1, p. 57-68, 2007.

BISPO JUNIOR, José P. Fisioterapia e saúde coletiva: desafios e novas responsabilidades profissionais. Ciência \& Saúde Coletiva, Rio de Janeiro, v. 15, supl. 1, p. 1627-1636, 2010.

BRASIL. Câmara dos Deputados.Projeto de Lei n. 3.256. Dispõe sobre a obrigatoriedade do atendimento fisioterapêutico pelas equipes do Programa de Saúde da Família. Comissão de Seguridade Social e Família, 2004a.<http:// www.camara.gov.br/proposicoesWeb/prop_mos trarintegra;jsessionid=D9800B3D50F6CD509 18F34C73DCF39EE. nodel codteor $=999633 \&$ filename $=$ Avulso $+-P L+4261 / 2004>$. Acesso em: 25 de ago. 2011.

BRASIL. Conselho Nacional de Educação. Câmara de Educação Superior. Resolução n. 4 CNE/CES de 19 fevereiro de 2002. Diretrizes Curriculares Nacionais do curso de Fisioterapia. Diário Oficial da União, Brasília, DF, 4 mar. 2002. Disponível em: <http://portal. mec.gov.br/cne/arquivos/pdf/CES042002. pdf>. Acesso em: 23 jul. 2010.

BRASIL. Ministério da Educação. A trajetória dos cursos de graduação na área da saúde: Instituto Nacional de Estudos e Pesquisas Educacionais Anísio Teixeira (Fisioterapia), 2006a. p. 201-240.
BRASIL. Ministério da Educação e do Desporto. Secretaria de Educação Superior. Edital n. 4/97 de 10 de dezembro de 1997. O MEC, por intermédio da SESu, torna público e convoca as instituições de ensino superior a apresentar propostas para as novas diretrizes curriculares dos cursos superiores, que serão elaboradas pelas Comissões de especialistas da SESu/MEC. Diário Oficial da União, Brasília, DF, seção III, p. 26.920, 12 dez. 1997. Disponível em: $<$ http://www.mec.gov.br/sesu/ftp /edital/e04/ doc $>$. Acesso em: 3 mar. 2011.

BRASIL. Ministério da Educação. Parecer CNE/ CES n. 1.210 de 07 de dezembro de 2001. Estabelece as Diretrizes Curriculares Nacionais dos Cursos de Graduação em Fisioterapia, Fonoaudiologia e Terapia Ocupacional. Conselho Nacional de Educação. Diário Oficial da União, Brasília: DF, 10 de dez 2001. Seção 1, p. 22. Disponível em: $<$ http://portal.mec. gov.br/cne/arquivos/pdf/pces1210_01.pdf $>$. Acesso em: 21 nov. 2010.

BRASIL. Ministério da Educação. Portaria Normativa MEC $\mathrm{n}^{\circ} 40$, de 12 de dezembro de 2007, republicada em 29 de dezembro de 2010. Dispõe sobre indicadores de qualidade e o Exame Nacional de Desempenho dos Estudantes. Diário Oficial da União, Brasília: DF, 12 de dez 2007. Seção 1, p. 23-31. Disponível em:http:// portal.mec.gov.br/index.php?option $=$ com docman \&view $=$ download \&alias $=16763$-portnorm-040-2007-seres\&Itemid=30192 Acesso em: 21 ago. 2012.

BRASIL. Ministério da Saúde. A construção do SUS: histórias da Reforma Sanitária e do Processo Participativo. Brasília: Ministério da Saúde, 2006b.

BRASIL. Ministério da Saúde; Ministério da Educação. Programa de Incentivo às Mudanças Curriculares nas Escolas Médicas. 2001. Disponível em: <http://portal.mec.gov.br/ sesu/arquivos/ pdf/inc.pdf $>$. Acesso em: 7 mar. 2013.

BRASIL. Ministério da Saúde. Portaria n. 154, de 24 de janeiro de 2008. Cria os Núcleos de 
Apoio à Saúde da Família (NASF). Brasília, 2008. Disponível em: <http://bvsms.saude.gov.br/ bvs/saudelegis/gm /2008/prt0154_24_01_2008. html>. Acesso em: 8 abr. 2013.

BRASIL. Ministério da Saúde. Saúde da Família: uma estratégia para reorientação do modelo assistencial. Brasília: Ministério da Saúde, 1998.

BRASIL. Ministério da Saúde. Secretaria de Gestão do Trabalho e da Educação na Saúde. Caminhos para a mudança da formação e desenvolvimento dos profissionais de saúde: diretrizes para a ação política para assegurar Educação Permanente no SUS. Brasília, Ministério da Saúde, 2003. 67 p.

BRASIL. Ministério da Saúde. Secretaria de Gestão do Trabalho e da Educação na Saúde. Política nacional de Educação Permanente em saúde. Brasília: Ministério da Saúde, 2009, 64p. (Série B. Textos Básicos de Saúde).

BRASIL. Ministério da Saúde. Secretaria de Saúde do município do Rio de Janeiro. O Programa Saúde da Família. Rio de Janeiro, 2014. Disponível em < http://www.rio.rj.gov. br/web/sms/clinicas-da-familia > . Acesso em: 15 jul. 2013.

CECCIM, Ricardo B.; FERLA Alcindo A. Notas cartográficas sobre a escuta e a escrita: contribuições à educação das práticas de saúde. In: PINHEIRO Roseni; MATTOS, Ruben A. (Org.). Construção social da demanda: direito à saúde, trabalho em equipe, participação e espaços públicos. Rio de Janeiro: IMS/UERJ, Cepesc, Abrasco, 2005. p.253-66.

COSTA, Marcelo V. et al. Pró-Saúde e PETSaúde como espaços de educação interprofissional. Interface: Comunicação, Saúde, Educação, Botucatu, v. 19, supl. 1, p. 709-720, 2015. Disponível em: <http://www.scielo.br/ scielo.php?script $=$ sci_arttext \& pid $=$ S1414$32832015000500709 \& \operatorname{lng}=$ en $\& n r m=i s o>$. Acesso em:6 mar. 2018.

ESCOREL, Sarah et al. O Programa de Saúde da Família e a construção de um novo modelo para a atenção básica no Brasil. Revista Pana- mericana de Salud Pública, Washington, v. 21, n. 2-3, p. 164-176, 2007.

FEUERWERKER Laura C.M.; SENA, Roseni R. Contribuição ao movimento de mudança na formação profissional em saúde: uma avaliação das experiências UNI. Interface: Comunicação, Saúde, Educação, Botucatu, v. 6, n. 10, p. 37-49, 2002.

FONSECA, Angélica F.; CORBO, Ana M. A. (org.). O território e o processo saúde-doença. Rio de Janeiro: EPSJV/Fiocruz, 2007.

FÓRUM DE PRÓ-REITORES DE EXTENSÃO DAS INSTITUIÇÕES PÚBLICAS DE EDUCAÇÃO SUPERIOR BRASILEIRAS (FORPROEX). Política Nacional de Extensão Universitária. Porto Alegre: Gráfica da UFRGS, 2012 (Coleção Extensão Universitária; v. 7).

FRASER, Márcia T. D.; GONDIM, Sônia M. G. Da fala do outro ao texto negociado: discussões sobre a entrevista na pesquisa qualitativa. Paideia, Ribeirão Preto, v. 14, n. 28, p. 139 152, 2004.

GALLO, Douglas L. L. A fisioterapia no Programa Saúde da Família: percepções em relação à atuação profissional e a formação universitária. 2005. 181 f. Dissertação (Mestrado em Saúde Coletiva) - Universidade Estadual de Londrina, Paraná, 2005.

GIL, Célia R. R. Formação de recursos humanos em saúde da família: paradoxos e perspectivas. Cadernos de Saúde Pública, Rio de Janeiro, v. 21, n. 2, p. 490-498, 2005.

GONDIM, Gracia M.M. et al. O território de saúde e territorialização. Cadernos de Saúde Pública, Rio de Janeiro, v. 17, n. 3, maiojun. 2001.

MINAYO, Maria C. S. O desafio do conhecimento: pesquisa qualitativa em saúde. 4. ed. São Paulo: Hucitec; Rio de Janeiro: Abrasco,1996.

MINAYO, Maria C. S. O desafio do conhecimento: pesquisa qualitativa em saúde. 8. ed. São Paulo: Hucitec, 2004. 
OLIVEIRA, Nara R.C. O currículo dos cursos de graduação em fisioterapia: a dimensão humana em análise. 2010. 99f. Dissertação (Mestrado em Educação) - Universidade do Vale do Itajaí, Santa Catarina, 2010.

PAIM, Jairnilson S. Saúde, politica e reforma sanitária: CEPS - Centro de Estudos e Projetos em Saúde. Salvador: Instituto de Saúde Coletiva-ISC, 2002.

REBELATTO, Jose R.; BOTOMÉ, Silvio P. Fisioterapia no Brasil: fundamentos para uma ação preventiva e perspectivas profissionais. 1 ed. São Paulo: Manole, 1999.

SERIANO, Kajena N.; MUNIZ, Vivianne R. C.; CARVALHO, Maria E. I. M. Percepção de estudantes do curso de fisioterapia sobre sua formação profissional para atuação na atenção básica no Sistema Único de Saúde. Fisioterapia e Pesquisa, São Paulo, v.20, n.3, p.250-255, set. 2013. Disponível em <http://www.scielo.br/ scielo.php ?script=sci_arttext\&pid=S180929502013000300009\&lng $=$ en $\& n r m=i s o>$. Acesso em: 8 set. 2017.

SIGNORELLI, Marcos C. et al. Um projeto político-pedagógico de graduação em fisioterapia pautado em três eixos curriculares.
Fisioterapia em movimento, Curitiba, v. 23, n. 2, p. 331-340, 2010.

SILVA, Daysi J.; ROS, Marco A. Inserção de profissionais de fisioterapia na equipe de Saúde da Família e Sistema Único de Saúde: desafios na formação. Ciência \& Saúde Coletiva, Rio de Janeiro, v. 12, n. 6, p. 1673-1681, dez. 2007. Disponível em: <http://www.scielo.br/ scielo.php?script $=$ sci_arttext\&pid $=$ S1413$81232007000600028 \& \operatorname{lng}=\mathrm{en} \& \mathrm{nrm}=$ iso $>$. Acesso em: 8 set. 2017.

SOUZA, Marcio C. et al. Formação acadêmica do fisioterapeuta para atenção básica. UNILUS Ensino e Pesquisa, São Paulo, v. 11, n. 23,p. 60, 2014.

STREIT, Derly S. et al. Educação Médica: dez anos de diretrizes curriculares nacionais. 1 ed. Rio de Janeiro: ABEM, 2012.

TRELHA, Celita S. et al. O fisioterapeuta no Programa de Saúde da Família em Londrina (PR). Revista Espaço para a Saúde, Londrina, v. 8, n. 2, p. 20-25, 2007.

Recebido em 29/11/2016. Aprovado em 19/09/2017. 